\title{
Heterogeneidad estructural y espacial de un bosque mixto dominado por Nothofagus dombeyi después de un disturbio parcial
}

\section{Structural and spatial heterogeneity of a mixed Nothofagus dombeyi-dominated forest stand after a partial disturbance}

\author{
DANIEL P. SOTO ${ }^{1, ~ *}$, CHRISTIAN SALAS ${ }^{2,3}$, PABLO J. DONOSO ${ }^{1} \&$ DANIEL UTEAU ${ }^{4}$ \\ ${ }^{1}$ Instituto de Silvicultura, Facultad de Ciencias Forestales y Recursos Naturales, Universidad Austral de Chile, Casilla 567, \\ Valdivia, Chile \\ 2 Departamento de Ciencias Forestales, Universidad de La Frontera, Casilla 54-D, Temuco, Chile \\ ${ }^{3}$ School of Forestry and Environmental Studies, Yale University, 360 Prospect Street, New Haven, CT 06511-2189, USA \\ ${ }^{4}$ Institut für Pflanzenernährung und Bodenkunde, Christian-Albrechts Universität zu Kiel, Hermann-Rodewald-Str. 224118 \\ Kiel, Germany \\ * Autor correspondiente: danielsoto@uach.cl
}

\begin{abstract}
RESUMEN
Los bosques dominados por Nothofagus dombeyi están entre las formaciones boscosas naturales más abundantes y productivas de la región templada de Sudamérica. En Chile son comunes los bosques secundarios dominados por $N$. dombeyi con una importante asociación con otras especies arbóreas subordinadas. Sin embargo, existen escasos estudios sobre su distribución diamétrica, patrón espacial y la asociación entre especies en estos bosques. En este trabajo se cuantifican dichos patrones y se prueba si existe una asociación espacial entre especies en un bosque estructuralmente complejo como resultado de un disturbio parcial. Para ello se estableció una parcela permanente de $70 \times 130 \mathrm{~m}$ (0,91 ha) en el predio Llancahue cercano a la ciudad de Valdivia $\left(39^{\circ} 48^{\prime} \mathrm{S}, 73^{\circ} 11^{\prime} \mathrm{O}\right.$ y $\left.150 \mathrm{msnm}\right)$. Se cuantificó la estructura y asimetría diamétrica por medio de la función de densidad de probabilidad de Weibull y el índice de asimetría, respectivamente. El patrón espacial (aleatorio, agrupado y regular) para el bosque y por especie, y la asociación espacial (indiferencia, repulsión y atracción) de $N$. dombeyi con las principales especies acompañantes, fueron evaluados con las funciones uni y bivariadas de Ripley. La autocorrelación espacial para el diámetro a la altura del pecho d fue evaluada por medio de semivariogramas. Se reportó que la distribución de tamaños del diámetro de los árboles (d) está influenciada por algunos individuos resistentes al disturbio y otros que regeneraron vegetativamente desde tocones, lo que es confirmado por el patrón espacial en agregados, y una consistente autocorrelación espacial para el tamaño de los árboles. Se comprobó que los patrones espaciales son intraespecíficos, y no influyeron sobre la asociación espacial de $N$. dombeyi con las especies subordinadas. Finalmente, se sugiere que los disturbios parciales provocan una transformación rápida de una estructura simple a una compleja a través de sucesión forestal.
\end{abstract}

Palabras clave: asociación espacial, dinámica de bosques, distribución horizontal, facilitación.

\begin{abstract}
The forests dominated by Nothofagus dombeyi are amongst the most abundant and productive forests in the South American temperate region. In the lowlands of Chile Nothofagus dombeyi-dominated secondary forests with their associated tree species are common. Their diameter distribution, spatial patterns and association between species have not been studied. In this work we quantify those patterns and test whether there is a spatial association between species in a structurally complex forest created after a partial disturbance. We established a 70 x $130 \mathrm{~m}$ plot $(0.91 \mathrm{ha})$ in the Llancahue property near the city of Valdivia $\left(39^{\circ} 48^{\prime} \mathrm{S}, 73^{\circ} 11^{\prime} \mathrm{W}\right.$ and 150 masl). We quantified the structure and the diameter asymmetry through the Weibull probability function and the asymmetry index, respectively. The spatial pattern (random, clustered and regular) of the forest and of each species, and the spatial association (indifference, repulsion and attraction) of $N$. dombeyi with the main species were evaluated with the uniand bivariate Ripley functions. The spatial autocorrelation for the diameter at breast height $\mathrm{d}$ was evaluated by means of semivariograms. This paper reports that the size distribution is influenced by some species that either resisted the disturbance or were highly resilient due to their regeneration strategies, which is confirmed by the spatial pattern in aggregates, and a strong spatial autocorrelation for the species. We found that the spatial patterns are intraspecific, and do not affect the spatial association between dominant and subordinated trees species. Finally, we suggest that partial disturbances cause a rapid transformation from a simple to a complex structure along forest succession.
\end{abstract}

Key words: facilitation, horizontal distribution, spatial association, stand dynamics. 


\section{INTRODUCCIÓN}

La teoría de la sucesión forestal explica que el reemplazo de especies es un proceso dinámico de la historia de vida de las especies en una comunidad o población, y determina su estructura presente y futura (Duncan 1991, Díaz \& Armesto 2007). Los patrones de reemplazo de especies en una comunidad ocurren debido a la competencia intra e interespecífica (proceso endógeno) que genera mortalidad de especies pioneras (generalmente especies sombra-intolerantes) y oportunidades para el ingreso de nuevas especies sucesionales (generalmente sombratolerantes) dependiendo si existe una relación de facilitación, inhibición o tolerancia entre las especies (sensu Conell \& Slatyer 1977). El establecimiento y sobrevivencia de las especies está determinado por las estrategias de asignación de recursos (luz, agua, nutrientes) y otras variables de la historia de vida de las especies, las cuales definen el éxito en distintas condiciones ambientales (Saldaña \& Lusk 2003, Soto \& Figueroa 2008), modificando los patrones demográficos de los bosques, como su estructura (e.g., distribuciones de tamaño), composición y distribución espacial.

La dinámica y sucesión forestal en rodales de Nothofagus Sudamericanos se han vinculado a disturbios catastróficos (sensu Veblen \& Ashton 1978, Veblen et al. 1980, 1981, 2004), y al igual que otros modelos de desarrollo de rodales en el mundo, se inician con la remoción "total" del área por parte de un determinado disturbio. Muchos trabajos sobre dinámica forestal en climas templados mencionan que son las especies arbóreas sombra-intolerantes las que colonizan un área "desnuda" perturbada, y se desarrollan hacia un punto de alta competencia intraespecífica y de autorraleo, que posteriormente facilita el reingreso de la vegetación de especies sombratolerantes $y$, consecuentemente, la diferenciación de copas hasta llegar a un estado de bosque adulto (Veblen \& Ashton 1978, Oliver \& Larson 1996, Veblen et al. 1980, 1981, 2004). Solo Veblen et al. (2004) mencionan la importancia sobre la sucesión forestal de árboles resistentes y resilientes al disturbio (i.e., disturbios parciales o crónicos) en ecosistemas templados sudamericanos, a pesar de no evaluar esto con datos empíricos. Es decir, si no se trata de disturbios de gran escala que desnudan un área, sino que de disturbios parciales que dejan legados vivientes que incluyen árboles y tocones de especies de reproducción vegetativa prolífica, es necesario preguntarse cuál es el efecto de estos legados sobre la sucesión forestal.

La evaluación de los patrones de disturbios y del desarrollo sucesional de los bosques generalmente se hace a través de la distribución de tamaños de árboles, en base al diámetro en el fuste a los $1.3 \mathrm{~m}$ (d) o altura total (h) (Veblen et al. 1980, 1981, Donoso et al. 1985, Baker et al. 2005, Goff \& West 1975 , Lorimer \& Krug 1983, Oliver \& Larson 1993, Baker et al. 2005, Zenner 2005). El estudio de los patrones espaciales de los árboles ayuda a la interpretación de la dinámica de bosques (Rozas 2006, Salas et al. 2006). Los métodos cuantitativos de análisis espacial tienen como objetivo detectar y describir los patrones de distribución (Rozas \& Camarero 2005, Duncan 1991, Salas et al. 2006), dando cuenta de la estructura espacial de una población, comunidad, o de cualquier fenómeno ecológico en un espacio determinado (Perry et al. 2006). En bosques, existe muchas veces una heterogeneidad espacial en la distribución de tamaños (e.g., d o h) tanto a nivel intraespecífico como interespecífico producto de fenómenos ecológicos que disminuyen la homogeneidad y/o regularidad inicial de las comunidades boscosas (Rozas \& Camarero 2005). Algunos factores contribuyentes a la pérdida de homogeneidad espacial son causados por las características de las especies (competencia por recursos, patrones diferenciales de regeneración, mortalidad, crecimiento diferencial) (Coomes \& Allen 2007, Wang et al. 2008), y otros por variables abióticas como variaciones topográficas o climáticas de gran y pequeña escala (Veblen et al. 2004), así como en la calidad de suelos (Moeur 1993) e interacciones comunitarias (Soto et al. 2009). Estos factores hacen que la distribución de tamaños de los árboles varíe en los diferentes tipos de bosques naturales en el mundo (Baker et al. 2005, Coomes \& Allen 2007).

Debido a que particularmente en zonas de menor altitud en la depresión intermedia de la zona centro-sur de Chile han sido frecuentes 
los disturbios parciales de bosques (por ramoneo, cortas selectivas y fuegos de baja intensidad), el presente trabajo tiene por objetivo evaluar el efecto de un disturbio de este tipo sobre la distribución de tamaños y espacial de un bosque dominado por $N$. dombeyi. Este estudio se hace mediante el estudio de la estructura, asimetría diamétrica, el patrón espacial univariante y la autocorrelación para el tamaño de los árboles del rodal, y para las principales especies en este bosque. Se evaluó también la asociación espacial bivariante entre el dosel de $N$. dombeyi respecto a las demás especies que participan en el bosque, con la finalidad establecer el vínculo espacial en un bosque estructuralmente complejo, y vincular así la coexistencia $N$. dombeyi con las otras especies semi y sombra-tolerantes ante un disturbio parcial.

\section{MÉTODOS}

\section{Área de estudio y mediciones}

El rodal de estudio se ubica en la cordillera de la Costa, en el predio forestal Llancahue (entre $39^{\circ} 48^{\prime} \mathrm{S}$ y $39^{\circ} 52^{\prime} \mathrm{S}$, y $73^{\circ} 11^{\prime} \mathrm{O}$ y $73^{\circ} 7^{\prime} \mathrm{O}$ ), a $150 \mathrm{~m}$ de altitud en una ladera media de exposición norte y pendiente media de $15 \%$. El clima según Koeppen es de costa occidental con influencia mediterránea, con una precipitación media anual de $2357 \mathrm{~mm}$, y una temperatura media de $11.9^{\circ} \mathrm{C}$ (Donoso et al. 2003). El suelo predominante es rojo arcilloso, originado por sedimentos plio-pleistocénicos depositados sobre esquistos metamórficos, de la serie Los Ulmos (Schlatter et al. 1995).

El bosque estudiado es mixto y multiestratificado, donde las principales especies son $N$. dombeyi, Laureliopsis philippiana, Eucryphia cordifolia, Aextoxicon punctatum, Weinmannia trichosperma, Gevuina avellana, y en menor medida se encuentran Laurelia sempervirens, Podocarpus saligna, Saxegothaea conspicua y Persea lingue (ver detalles del bosque en Donoso et al. 2003).

Se midieron todos los árboles vivos con un d mayor a $5 \mathrm{~cm}$ en una parcela de muestreo de $130 \times 70 \mathrm{~m}(0,91$ ha). Cada árbol fue localizado espacialmente mediante 91 cuadrículas de $10 \times 10 \mathrm{~m}$, con coordenadas cartesianas (x, y) de cada individuo (Fig. 1).

\section{Análisis estadístico}

Se analizó cuantitativamente a) la estructura diamétrica, b) los patrones espaciales uni y bivariante, y c) la correlación de tamaños en el espacio. La estructura diamétrica del bosque fue analizada mediante el ajuste de la función de densidad de probabilidad (fdp) de Weibull a los datos de d de árboles sin segregación de especies y para cada una de ellas. El modelo de Weibull permite representar distribuciones simétricas, así como asimétricas de diversas formas dependiendo del valor de los parámetros. Se empleo la siguiente reparametrización del modelo de Weibull;

$$
f(d)=\frac{\alpha}{\beta}\left(\frac{x}{\beta}\right)^{\alpha-1} \exp \left[-\left(\frac{x}{\beta}\right)^{\alpha}\right]
$$

para $\mathrm{x} \geq 0$, donde $\alpha$ y $\beta$ son parámetros de forma y de escala, respectivamente. Una de las ventajas del uso del modelo de Weibull para modelar distribuciones diamétricas es la interpretación de sus parámetros, ya que tal como lo destaca Bailey \& Dell (1973) y Lorimer $\&$ Krug (1983), valores de $\alpha$ inferiores a 1 describen una curva monotónica y descendente (J-inversa), mientras que valores mayores a 1 y menores a 3.6 describen una curva unimodal con asimetría positiva (como la descrita por la fdp Normal), y valores iguales a 3.6 representan una función aproximadamente normal, y finalmente valores mayores a 3.6 describen una curva normal con asimetría negativa. El modelo de Weibull (Eq. 1) fue ajustado mediante el método de máxima verosimilitud, para lo cual se empleó el programa estadístico $\mathrm{R}$ (R Development Core Team 2009). La asimetría de las distribuciones diamétricas fue caracterizada mediante el índice de simetría $\left(\mathrm{S}_{\mathrm{k}}\right)$.

Los patrones de distribución espacial fueron analizados tanto en forma uni y bivariante mediante la función K de Ripley (Ripley 1976, 1981), la cual emplea la distancia entre puntos obtenida a través de las coordenadas espaciales de los árboles de la parcela. Dicha función ha sido empleada con éxito en diversos estudios ecológicos tanto en matorrales (Haase 1995) como en bosques (Mouer 1993, He \& Duncan 2000, Salas et al. 2006, Fajardo \& Alaback 2007), por lo cual se optó por emplear esta función también en este estudio. La función K de Ripley (Ripley 1976, 1981) corresponde a la siguiente expresión;

$$
K(t)=\frac{A}{n^{2}} \sum_{i=1}^{n} \sum_{j=1}^{n} \frac{1}{w_{i j}} I_{t}\left(u_{i j}\right),
$$

donde, $\mathrm{n}$ es el número de puntos en la unidad de muestreo (parcela), A es el área de la unidad de muestreo en $\mathrm{m}^{2}, \mathrm{u}_{\mathrm{ij}}$ es la distancia entre el i-ésimo árbol sujeto y el j-esimo árbol, expresada en $\mathrm{m}, \mathrm{w}_{\mathrm{ij}}$ es el factor de corrección del efecto borde para los respectivos puntos $\mathrm{i}-\mathrm{j}$. $\mathrm{I}_{\mathrm{i}}$ es un indicador si el punto está en el radio de búsqueda t. Se empleó el factor de corrección propuesto por Haase (1995). Posteriormente, se estabilizó la varianza y linealizó la función mediante una transformación de la función $\hat{\mathrm{K}}(\mathrm{t})$ como sigue;

$$
L(t)=\sqrt{K(t) / \pi},
$$

si $\hat{L}(t)$ es cero, mayor a cero, o menor a cero, se interpreta que el patrón espacial es aleatorio, agrupado, o regular, respectivamente (Mouer 1993; Salas et al. 2006). Los intervalos de confianza para el patrón de distribución aleatorio fueron confeccionados a un nivel de significancia del $5 \%$ mediante simulaciones Monte Carlo (más detalles respecto a esto y referencias son dados en Salas et al 2006). La función (3) fue calculada para el bosque total y para cada especie por separado. 

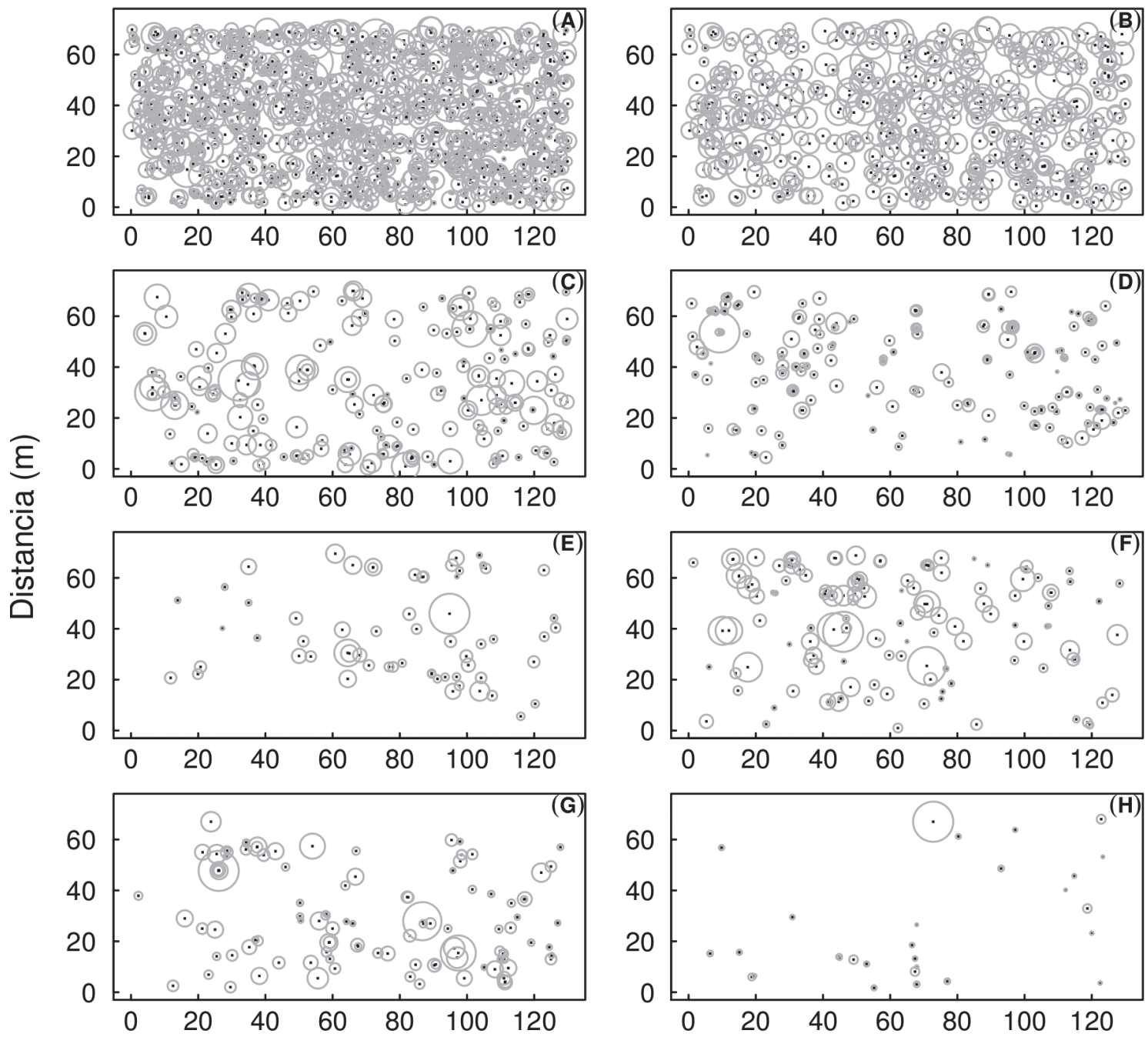

\section{Distancia $(m)$}

Fig. 1: Mapa de localización espacial de árboles en una parcela permanente de 70 x $130 \mathrm{~m}$ en el bosque de Llancahue, Chile. Figuras muestran los datos para A) todas las especies, B) N. dombeyi, C) E. cordifolia, D) G. avellana, E) L. sempervirens, F) L. philippiana, G) A. punctatum, and H) P. saligna. Círculos son proporcionales al d x 100 en todos los árboles muestreados.

Location map of live trees in a 70 x 130 m permanent sample plot at Llancahue Forest, Chile. Figures show data for A) all species, B) N. dombeyi, C) E. cordifolia, D) G. avellana, E) L. sempervirens, F) L. philippiana, G) A. punctatum, and H) $P$. saligna. Circles are proportional to $\mathrm{d} \times 100$ of each sampled tree.

Se empleó la función bivariante de Ripley, $\hat{\mathrm{K}}_{12}(\mathrm{t})$, para analizar la interacción ó asociación espacial de los distintos grupos de análisis, en nuestro trabajo de $N$. dombeyi respecto a las especies subordinadas. Esta función indica el tipo, intensidad y rango de distribución espacial que presentan dos grupos de puntos (Rozas 2006, Rozas \& Camarero 2005, Salas et al. 2006). Esta función se calcula para dos grupos de análisis, i.e. 1 y 2 , calculando distancias (ecuación 1 ), pero tanto desde el grupo 1 al 2, como del 2 al 1, para luego ponderar estas dos funciones, y obtener una función final denomina $\hat{\mathrm{K}}_{12}(\mathrm{t})$, la cual es linealizada de igual manera $\left(\hat{\mathrm{L}}_{12}(\mathrm{t})=\sqrt{\left.\hat{\mathrm{K}}_{12}(\mathrm{t}) / \pi\right)}\right.$ a la versión univariante (Salas et al. 2006). Cuando el gráfico de $\hat{\mathrm{L}}_{12}(\mathrm{t})$ versus $\mathrm{t}$ es significativamente mayor a cero existe atracción entre las especies, y repulsión entre especies cuando es significativamente menor a cero (Moeur 1993, Rozas \& Camarero 2005, Salas et al. 2006). Los intervalos de confianza fueron establecidos mediante simulaciones de Monte Carlo, de igual manera que para el caso univariante. El ajuste de la función de Ripley uni y bivariante fueron realizados en el paquete estadístico SpPack (Perry 2004). 
Para el análisis de la correlación espacial de tamaños se empleó d como variable respuesta para la evaluación de la correlación espacial entre tamaños de árboles. Para esta evaluación, normalmente se ocupan correlogramas y semivariogramas. Un correlograma es un gráfico que relaciona el coeficiente de correlación lineal entre los valores de la variable respuesta calculado para árboles dentro de una misma clase de distancia y la siguiente, versus la distancia. Un semivariograma entretanto, es la varianza de la variable respuesta entre puntos a una distancia determinada versus distancia. El semivariograma poblacional corresponde a la expresión matemática (e.g., Waller \& Gotway 2004)

$$
\gamma(t)=\frac{1}{2} \operatorname{Var}[Z(s)-Z(s+t)],
$$

donde, Var es el operando de la varianza estadística, $Z$ (s) es la observación de la variable $Z$ (en este caso diámetro del árbol) en la localización espacial s y t distancia entre observaciones. La curva descrita por la función (4), semivariograma, tiene en general una forma típica que comienza en cero, y si las observaciones cercanas son más parecidas que observaciones lejanas, la curva se incrementa a medida que la separación también se incrementa hasta alcanzar una distancia donde la curva se estabiliza (Schabenberger \& Pierce 2002, Waller \& Gotway 2004). Un aumento de la variabilidad (i.e., semivariograma) implica una disminución de la correlación espacial. La estimación de los semivariogramas a partir de los datos es más segura que la estimación de los correlogramas, ya que la estimación de los primeros no requiere la estimación de la media (Waller \& Gotway 2004). Por esta razón, se ajustaron semivariogramas en vez de correlogramas. Se calcularon los semivariogramas empíricos para todos los árboles y para cada especie presente por separado, así como también se ajustaron diferentes modelos de semivariogramas (Exponencial, Esférico, y Gaussiano). La máxima distancia empleada en la construcción de los semivariogramas fue de $35 \mathrm{~m}$. Los cálculos fueron realizados empleando el paquete geoR del software estadístico R (Ribeiro \& Diggle 2001).

\section{RESULTADOS}

\section{Estructura diamétrica}

El ajuste del modelo de Weibull fue estadísticamente significativo para todos los grupos funcionales analizados, así como también para sus parámetros estimados $(\mathrm{P}<$ $0.001)$, mayores detalles ver Salas \& Soto (2010). Los valores estimados para el parámetro de forma $\alpha$ fueron altos (> 1.5) para la comunidad (todas las especies), y para $N$. dombeyi, L. sempervirens y E. cordifolia, siendo el valor más alto aquel para $N$. dombeyi (Fig. 2). Por otro lado, G. avellana, P. saligna, $L$. philippiana y $A$. punctatum presentaron un parámetro estimado de forma $\alpha$ cercano a 1 , donde $A$. punctatum tuvo el valor menor (Fig. 2). El índice de simetría $S_{\mathrm{k}}$ muestra una curva normal con cierto desplazamiento hacia la izquierda con valores de asimetría positivos y bajos para la comunidad, y para $N$. dombeyi, $E$. cordifolia y L. philippiana. Por otro lado, se observaron valores altos de $\mathrm{S}_{\mathrm{k}}$ con asimetría hacia la izquierda de manera ascendente para A. punctatum, L. sempervirens, G. avellana y $P$. saligna (Fig. 2).

\section{Patrones espaciales univariados}

$N$. dombeyi se distribuyó espacialmente de forma aleatoria a lo largo de los $35 \mathrm{~m}$ de radio analizados (Fig. 3). En cambio, G. avellana y A. punctatum presentaron una distribución espacial en agregados para el mismo radio. En tanto la comunidad, $L$. sempervirens y $L$. philippiana, presentaron una distribución mayoritariamente en agregados (Fig. 3). E. cordifolia presentó un patrón en agregados hasta distancias de $16 \mathrm{~m}$, y a partir de esta hasta los $35 \mathrm{~m}$ presentó una distribución aleatoria; $P$. saligna presentó un patrón mixto intermitente entre aleatorio y agrupado, pero con diferencias significativas respecto a un patrón aleatorio (Fig. 3).

\section{Patrones espaciales bivariados}

El bosque dominado por $N$. dombeyi no afectó significativamente la distribución espacial de E. cordifolia $(\mathrm{P}=0.202), P$. saligna $(\mathrm{P}=0.080)$, G. avellana $(\mathrm{P}=0.143)$, y $A$. punctatum $(\mathrm{P}=$ $0.336)$, lo que ilustra que estas especies presentan una total indiferencia espacial respecto a la dominancia de $N$. dombeyi (Fig. 4). L. sempervirens tuvo un patrón mayoritariamente indiferente, pero en distancias cortas presentó atracción con $N$. dombeyi $(\mathrm{P}=0.028$; primeros $3 \mathrm{~m})$. Por otra parte, L. philippiana presentó un patrón espacial mayoritariamente indiferente con $N$. dombeyi, pero a distancias intermedias registró repulsión respecto a esta especie $(\mathrm{P}=0.004$; entre los 12 y $18 \mathrm{~m}$ ).

\section{Autocorrelación del tamaño en el espacio}

El modelo exponencial se ajustó bien a los semivariogramas empíricos para casi todas las especies (Fig. 5A). Sin embargo, existieron 

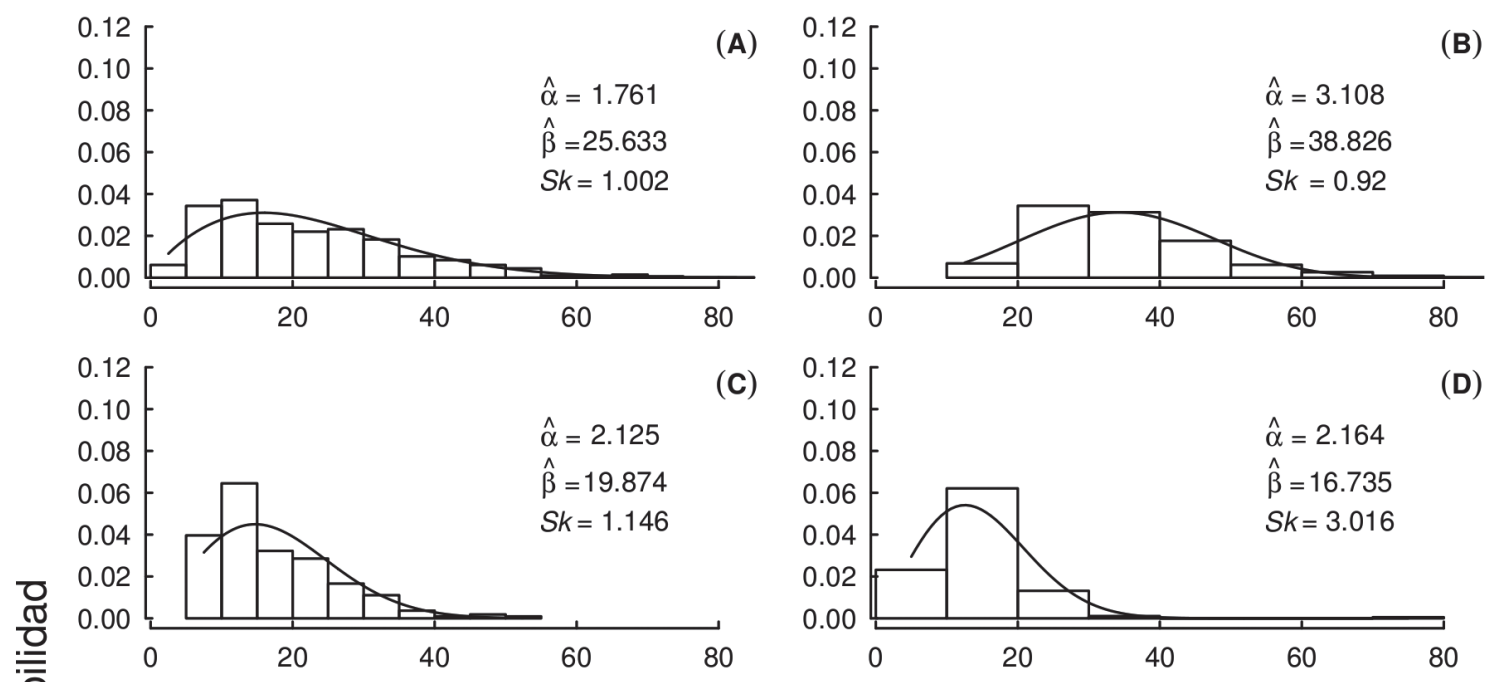

(C)
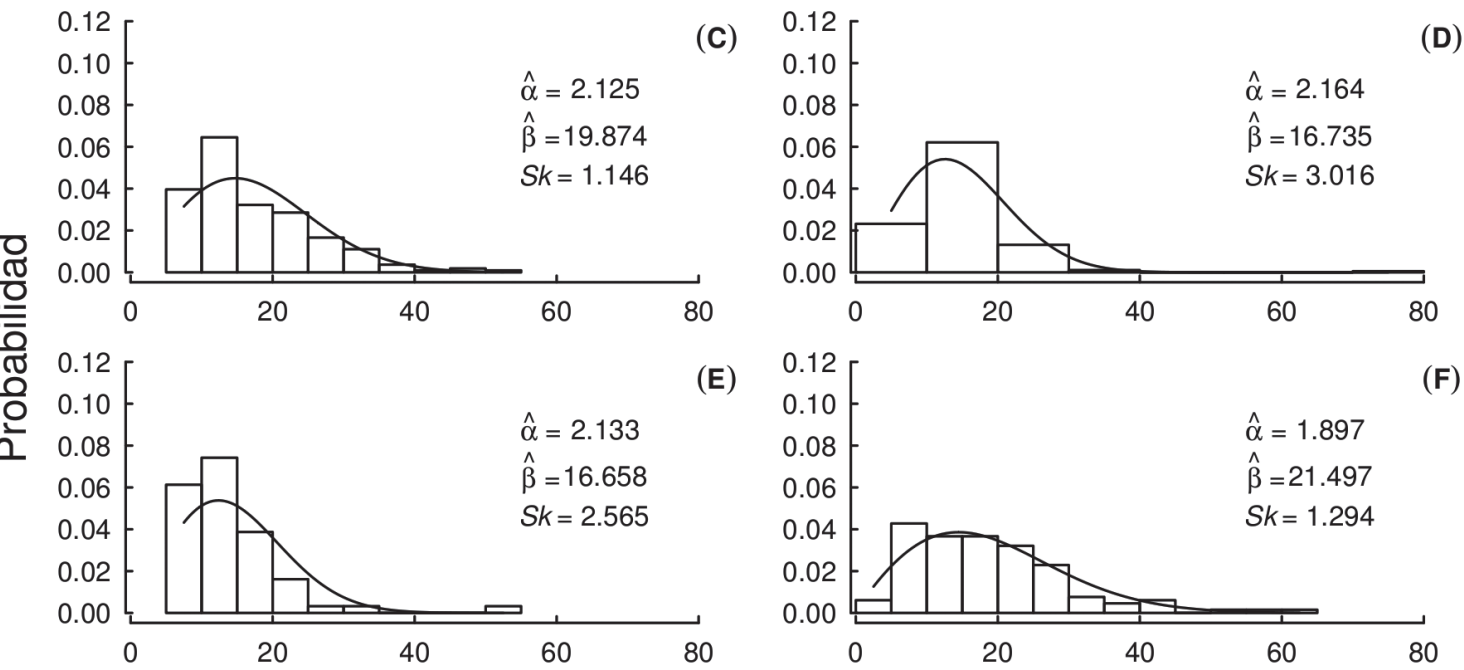

(E)
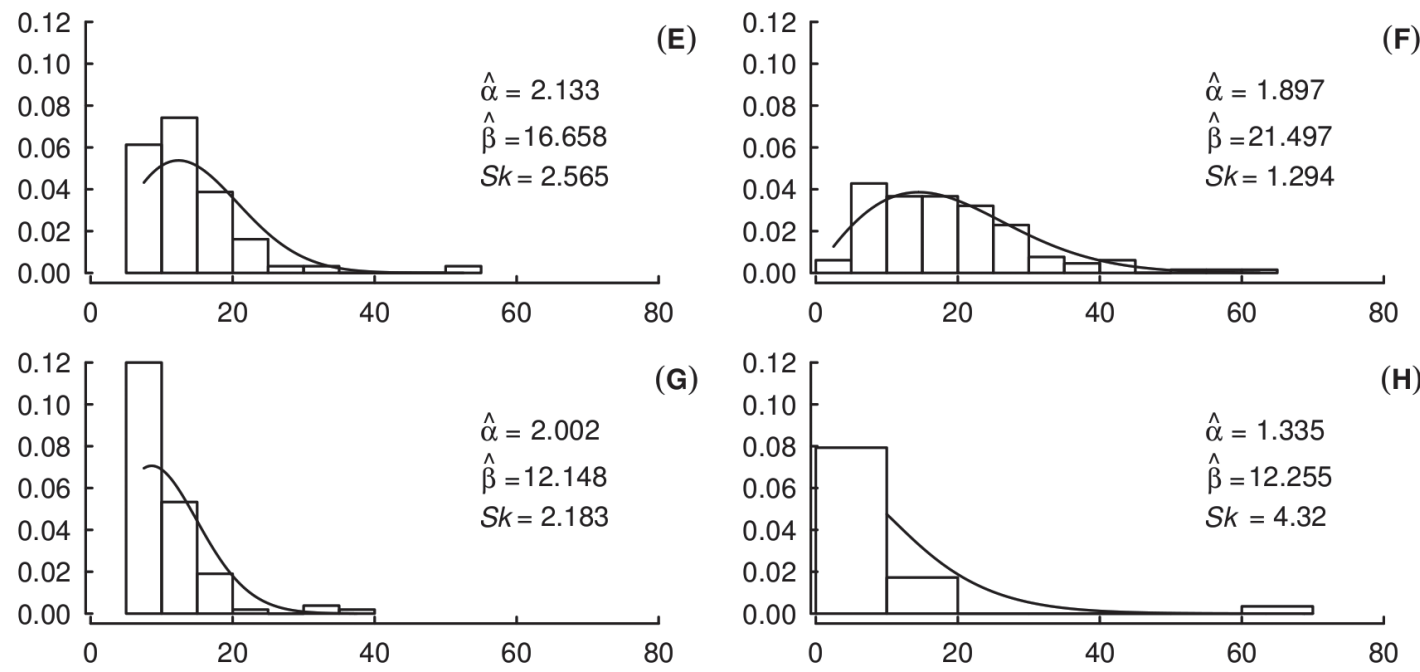

(G)

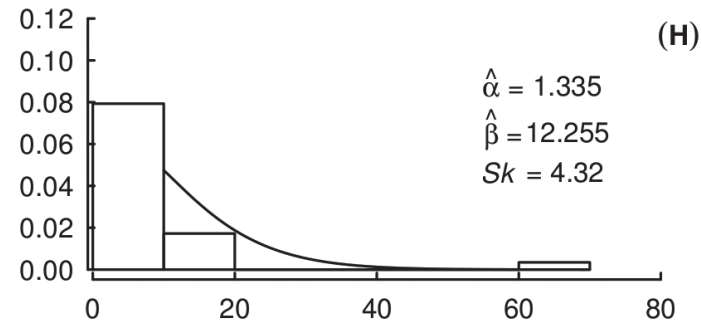

Diámetro $(\mathrm{cm})$

Fig. 2: Distribución diamétrica del bosque total (i.e., todas las especies) y por especies. La curva representa el ajuste de la función de Weibull $\alpha=$ parámetro de escala, $\beta=$ parámetro de forma y $\mathrm{S}_{\mathrm{k}}=$ coeficiente de asimetría. Todos los ajustes fueron altamente significativos $(\mathrm{P}<0.0001)$. A) todas las especies, B) $N$. dombeyi, C) E. cordifolia, D) G. avellana, E) L. sempervirens, F) L. philippiana, G) A. punctatum, y H) P. saligna.

Diameter distribution by species and Weibull function fit. $\alpha=$ scale parameter, $\beta=$ form parameter and sk $=$ Skewness. All fits were highly significative $(\mathrm{P}<0.0001)$. A) all species, B) N. dombeyi, C) E. cordifolia, D) G. avellana, E) L. sempervirens, F) L. philippiana, G) A. punctatum, and H) P. saligna.

problemas de convergencia en el ajuste de los modelos para los semivariogramas de $A$. punctatum y P. saligna (Figs. 5G y 5H), por lo cual se optó por no generalizar nuestros resultados para dichas especies. La autocorrelación espacial de d decrece notoriamente con la distancia, considerando a toda la comunidad (Fig. 5A) o cada una de las especies por separado. Aunque en términos generales la variabilidad espacial aumenta con la distancia (i.e., decrece la autocorrelación), esta no es tan aparente para $N$. dombeyi (Fig. 5B). La autocorrelación espacial fue relativamente fuerte en los primeros $10 \mathrm{~m}$ para E. cordifolia y L. philippiana (Figs. 5C y 5E, respectivamente), y aunque $L$. semprevirens mostró la misma tendencia, su ajuste fue pobre $\mathrm{y}$ no es posible inferir esto con certeza. 


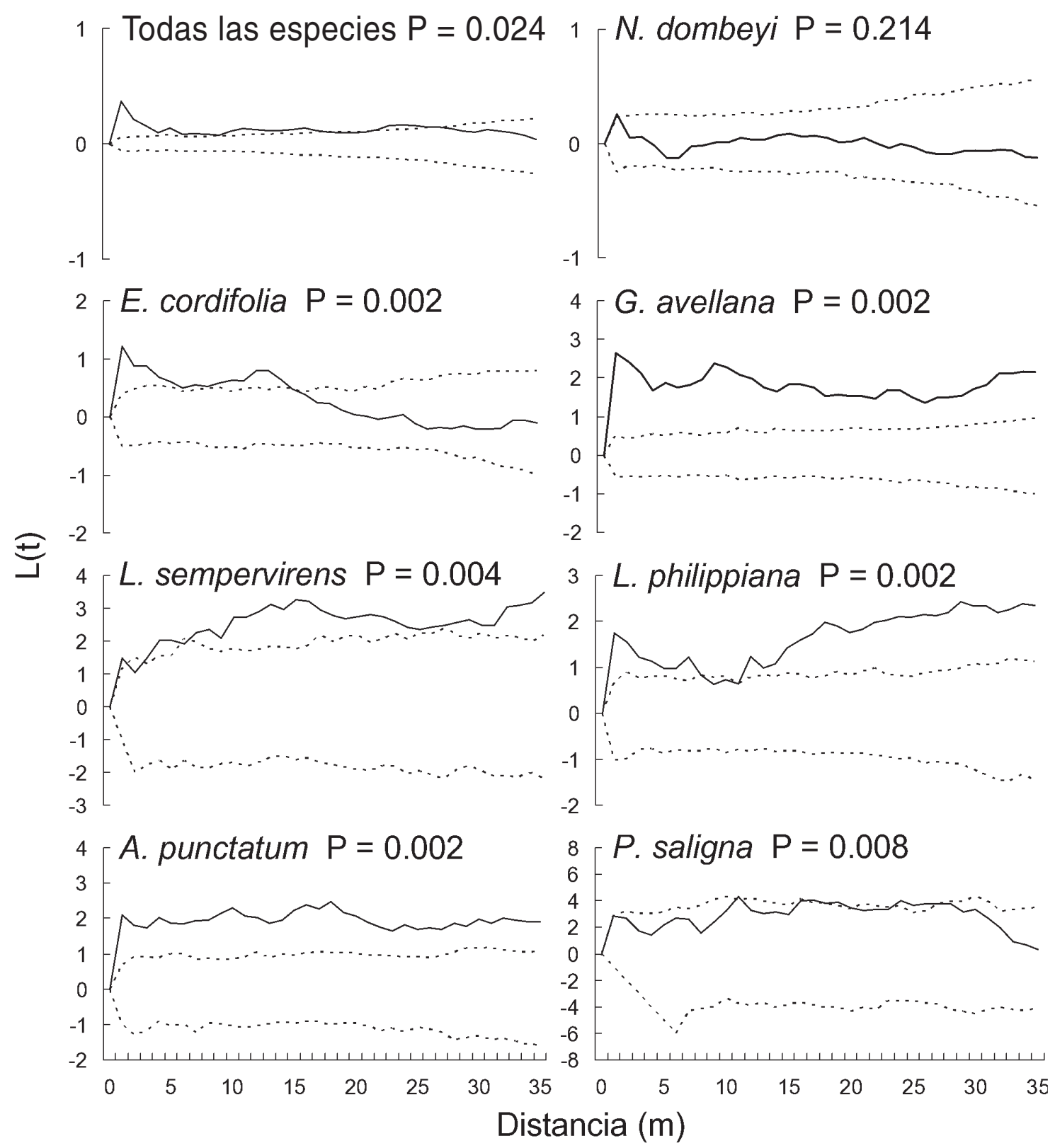

Fig. 3: Patrones espaciales univariados de la función $\hat{\mathrm{K}}(\mathrm{t})$ de Ripley para el bosque de Llancahue. La muestra estadística $\hat{L}(\mathrm{t})$ es mostrada por la línea sólida, así como $95 \%$ de intervalos de confianza para el proceso espacial aleatorio está dado por línea punteada.

Univariate spatial patterns of the Ripley's $\hat{K}(t)$ function for the Llancahue forest. The sample statistics $\hat{L}(t)$ is shown as a solid line, whereas the pointwise $95 \%$ confidence interval for a random spatial process is given as dotted lines.

\section{DISCUSIÓN}

Los resultados muestran que el bosque analizado se encuentra en una fase de "madurez" (asimetría hacia la izquierda), confirmada por un patrón unimodal para $N$. dombeyi, E. cordifolia y L. sempervirens, bajo las cuales existe una alta densidad de especies sombra-tolerantes con asimetría a la izquierda (Fig. 2). Aparte de la estratificación entre estos dos grupos funcionales (dominantes y subordinados), también se reportó una estratificación de tamaños para las especies sombra-tolerantes y algunas semitolerantes, existiendo dos grupos de clases de tamaño: uno con gran presencia de individuos en las 

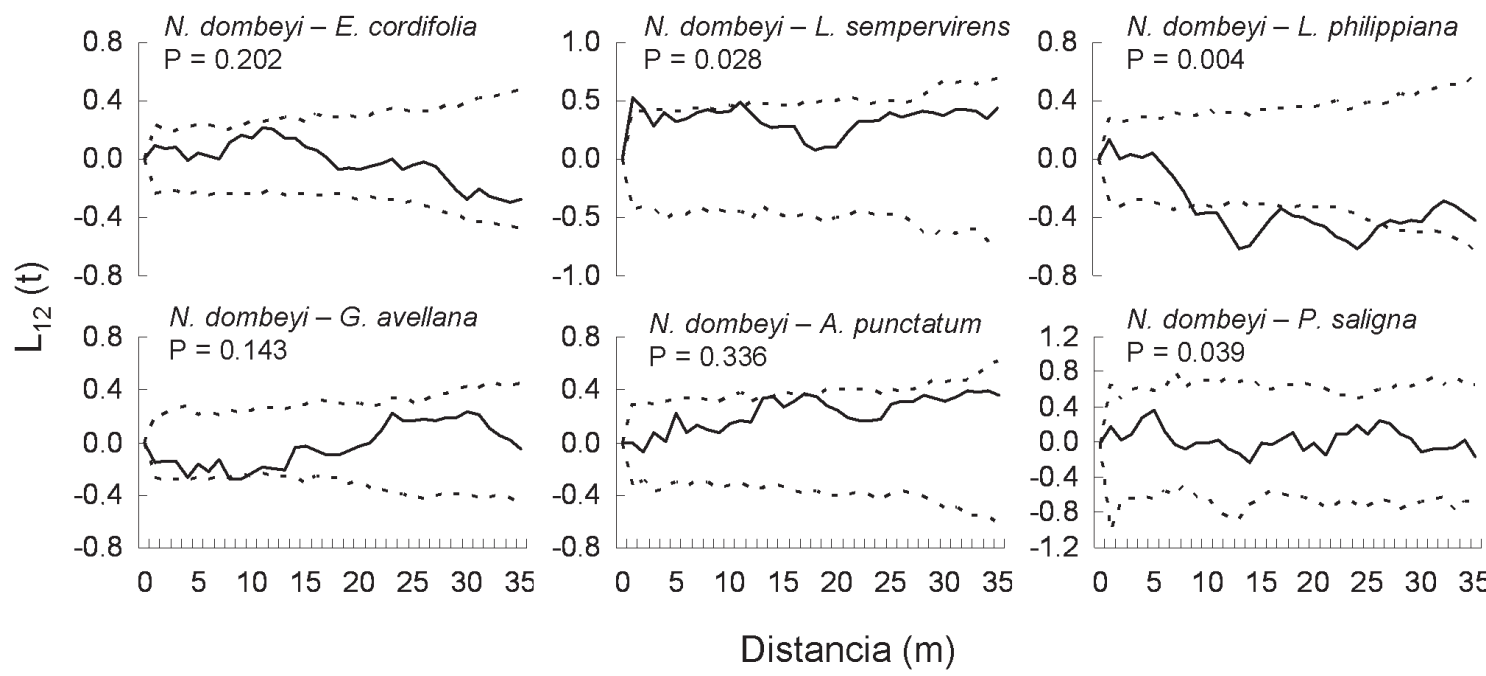

Fig. 4: Asociación espacial bivariada de la función $\hat{\mathrm{K}}_{12}(\mathrm{t})$ de Ripley para $N$. dombeyi con las principales especies arbóreas del bosque de Llancahue. La muestra estadística $\hat{\mathrm{L}}_{12}(\mathrm{t})$ se muestra con la línea sólida, así como del $95 \%$ de intervalos de confianza para el proceso de indiferencia espacial esta dado por líneas punteada.

Bivariate spatial association of the Ripley's $\hat{\mathrm{K}}_{12}(\mathrm{t})$ function for $N$. dombeyi with the main associate trees species at the Llancahue forest. The sample statistics $\hat{\mathrm{L}}_{12}(\mathrm{t})$ is shown as a solid line, whereas the pointwise $95 \%$ confidence interval for an indifference spatial process is given as dotted lines.

clases diamétricas inferiores, y otro con algunos individuos en las clases diamétricas mayores (e.g., E. cordifolia, L. sempervirens, G. avellana, L. philippiana y P. saligna).

En ecología forestal un supuesto común es que los árboles de pequeño tamaño presentan un patrón espacial agrupado, y los árboles de gran tamaño se distribuyen de forma aleatoria (Szwagrzyk \& Czerwczak 1993, Aldrich et al. 2003, Rozas 2006), lo cual es respaldado por nuestros resultados. El proceso de cambio de un patrón agrupado (bosque joven) hacia uno aleatorio (bosque maduro y/o adulto) (sensu Szwagrzyk \& Czerwczak 1993, Aldrich et al. 2003), es provocado por el proceso de mortalidad asociada a la competencia por recursos (Kenkel 1988, Leps \& Kindlmann 1987). Los patrones espaciales de las especies son divergentes entre $N$. dombeyi y $E$. cordifolia y las subordinadas G. avellana, $L$. sempervirens, L. philippiana, A. punctatun y $P$. saligna, predominando el patrón aleatorio para los árboles dominantes con grandes diámetros, y un patrón en agregados para especies subordinadas pertenecientes a las clases de diámetro menores, resultados similares a los reportados por Mouer (1993), Ward et al. (1996), y Aldrich et al. (2003). Estos patrones espaciales son corroborados por la autocorrelación espacial por medio de semivariogramas, con valores altos para los diámetros menores y bajos para los mayores (Fig. 5).

El estudio reporta un patrón espacial aleatorio para $N$. dombeyi, el que ha sido también reportado por Salas et al. (2006) para un bosque adulto dominado por Nothofagus obliqua (c.a. 450 años). De igual manera, $E$. cordifolia muestra un patrón agregado a distancias pequeñas y aleatorio a distancias mayores, como $N$. dombeyi. Esto indicaría una pérdida de autocorrelación a diámetros mayores de manera temprana, lo que podría estar provocado por mortalidad intraespecífica (Fig. 2). Por otra parte, las especies subordinadas presentan un patrón en agregados, lo cual es típico para este tipo de especies (Aldrich et al. 2003, Salas et al. 2006) .

$N$. dombeyi presenta mayoritariamente una asociación espacial indiferente con respecto a las restantes especies (Fig. 4). Se puede inferir que durante la fase de mortalidad intraespecífica de individuos dominantes de $N$. dombeyi se crearían condiciones heterogéneas de disponibilidad de recursos (luz, agua, nutrientes), las que provocarían condiciones propicias para la regeneración agrupada y continua de la especies subordinadas bajo el 


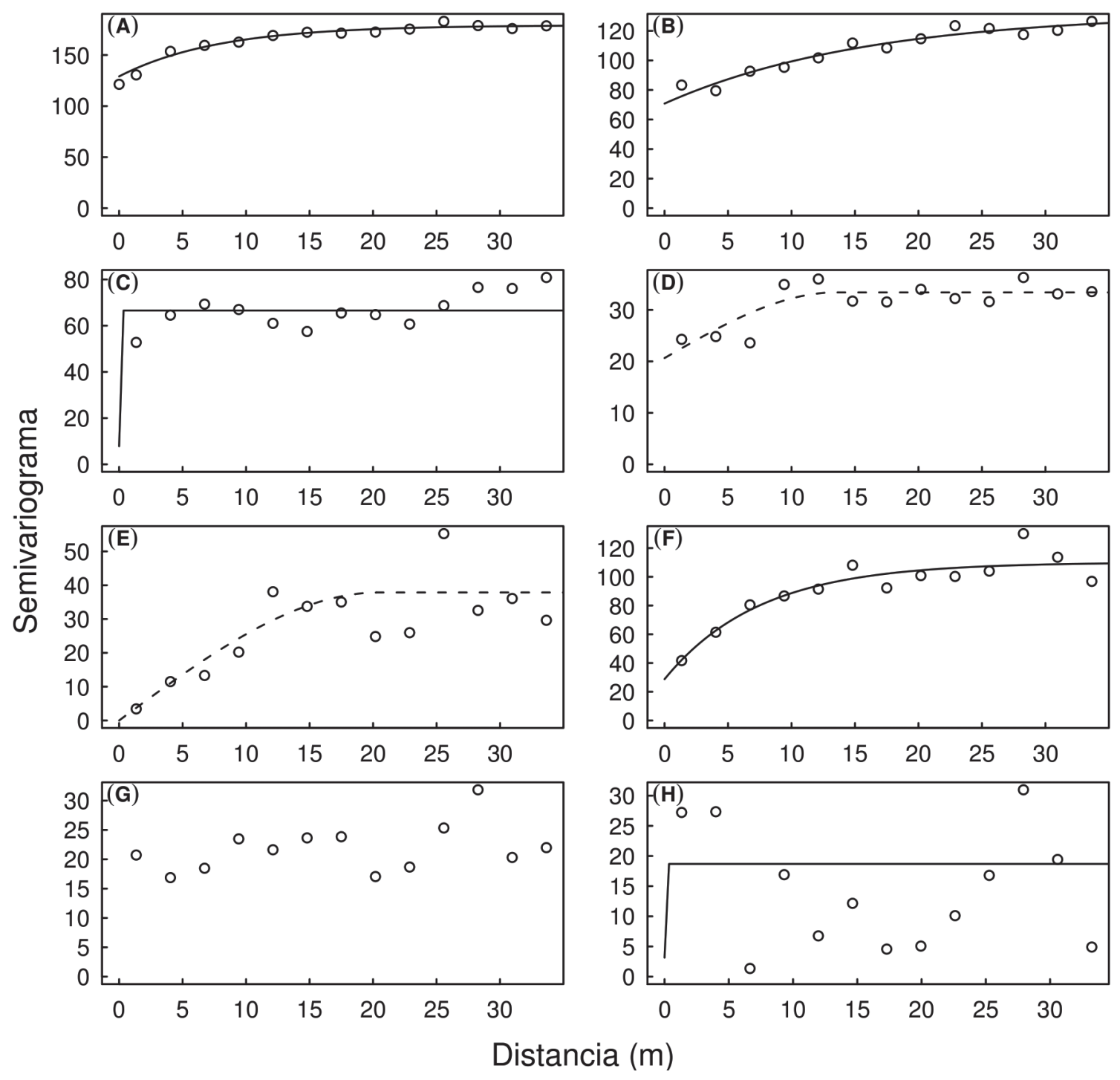

Fig. 5: Semivariogramas empíricos (puntos) y paramétricos (Exponencial: línea sólida; Esférico: línea discontinua; Gausiana: línea punteada) para el d de los árboles de A) todas las especies, B) $N$. dombeyi, C) $E$. cordifolia, D) G. avellana, E) L. Sempervirens, F) L. philippiana, G) A. punctatum, y H) P. saligna.

Empirical (dots) and parametric (Exponential: solid line; Spherical: dashed line; Gaussian: dotted line) semivariogram models for d for trees of A) all species, B) $N$. dombeyi, C) E. cordifolia, D) G. avellana, E) L. Sempervirens, F) L. philippiana, G) A. punctatum, and H) P. saligna.

estrato semiabierto y alto de $N$. dombeyi. De esta manera, se puede afirmar que el desarrollo del bosque analizado responde a un proceso endógeno e intraespecífico, el cual provoca facilitación para el establecimiento de las especies subordinadas. Este patrón ha sido también reportado en otros bosques de latifoliadas en climas templados (e.g., Aldrich et al. (2003) en Estados Unidos, Chen \& Bradshaw (1999) en China, y Fajardo \& Alaback (2005) en Chile).
El presente estudio ilustra el efecto de los disturbios parciales sobre los patrones estructurales, espaciales y la asociación de las especies, particularmente en cuanto a cómo estos pueden ayudar a una rápida estratificación del tamaño de los árboles (d). La existencia de árboles en pie que sobrevivieron el disturbio (particularmente $N$. dombeyi), asociada a la posible rápida proliferación vegetativa de $L$. sempervirens, $L$. philippiana, y algunos individuos de $E$. 


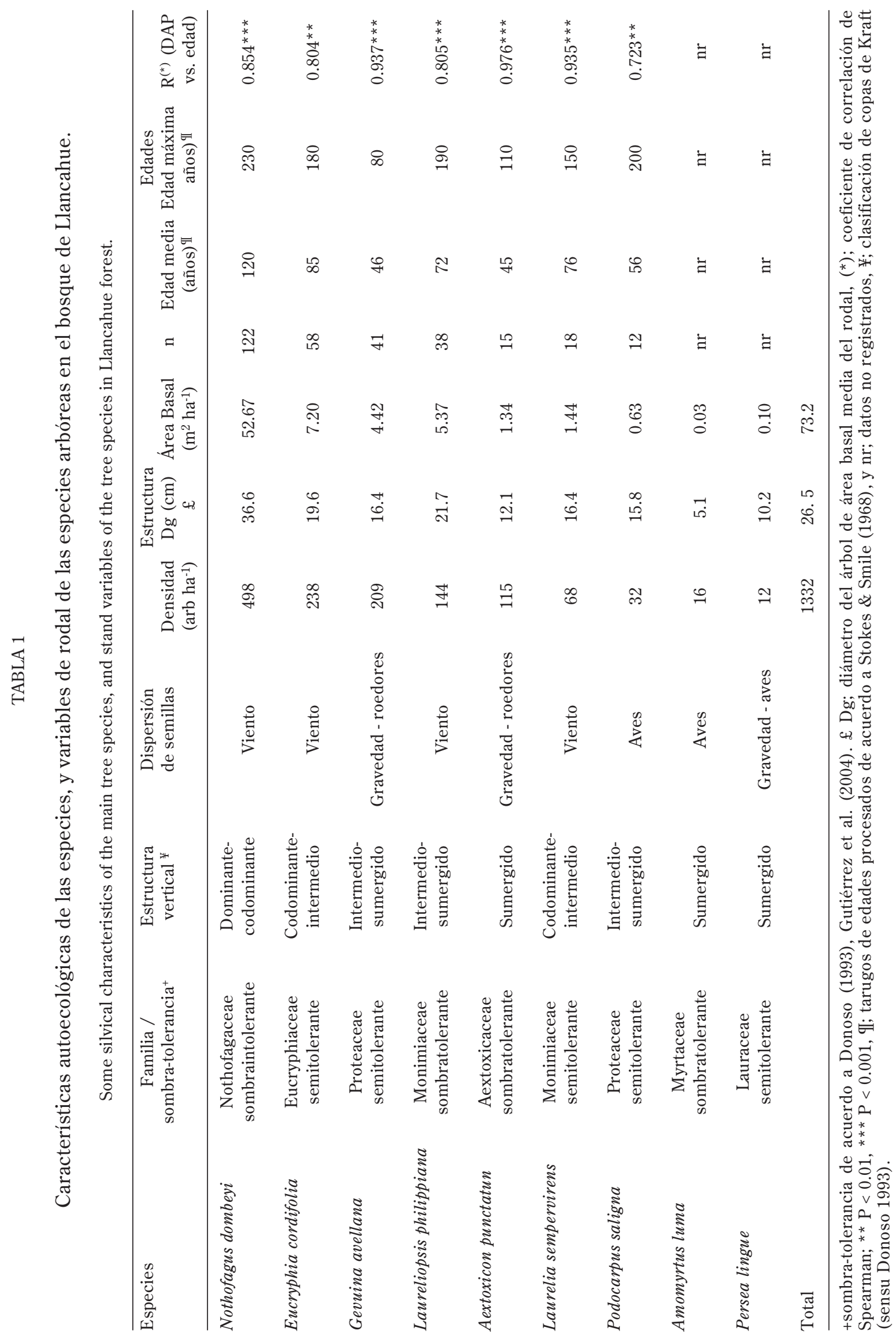


cordifolia, que regeneran masivamente a partir de rebrotes de tocones y raíces (Escobar et al. 2006), serían causas fundamentales para generar los patrones de distribución espacial y de estratificación observados. Esta rápida estratificación del tamaño de los árboles $(<200$ años $)$ se puede apreciar a través de la distribución de tamaños por medio de una curva sigmoídea rotada para todas las especies (Zenner 2005). Espacialmente se registró una completa aleatoriedad para $N$. dombeyi (Fig. 3) a pesar de tener unos 200 años menos que $N$. obliqua en el estudio de Salas et al. (2006). Se podría inferir que el bosque analizado presenta una rápida transformación desde un bosque joven hacia uno maduro multiestratificado debido al establecimiento de especies semi y sombratolerantes de forma más o menos coetánea (entre 150 a 230 años), generando en definitiva una estructura compleja (Zenner 2005)

El bosque analizado presenta procesos de competencia y mortalidad intraespecífica de $N$. dombeyi, fenómeno que no influye sobre el patrón de asociación espacial bivariante de las especies subordinadas (Fig. 4). Esto promueve la coexistencia de especies sombraintolerantes con respecto a las semi y sombratolerantes (Lusk 1995, Christie \& Armesto 2003, Soto \& Donoso 2006). De esta manera, la coexistencia del bosque analizado es el resultado del comportamiento diferencial de los distintos grupos funcionales ante la disponibilidad de recursos (Pickett et al. 1987, Saldaña \& Lusk 2003). La indiferencia espacial entre los grupos funcionales ha sido reportada para bosques secundarios de Nothofagus spp. por Lusk \& Ortega (2004), y para un bosque adulto de $N$. dombeyi en los Andes chilenos por Donoso \& Lusk (2007), en ambos casos reportándose que un dosel dominado por Nothofagus no afecta el establecimiento y la demografía individual de las especies subordinadas.

\section{CONCLUSIONES}

El presente estudio analizó cuantitativamente la importancia de especies resistentes y resilientes al disturbio sobre la estructura, patrón y asociación espacial de un rodal generado luego de este disturbio. Sobre 1o anterior, la estructura diamétrica está fuertemente influenciada por dichos árboles, y estos transforman el bosque desde una estructura simple hacia una multiestratificada de manera rápida en el proceso sucesional (c.a. 200-250 años). Se sugiere que diámetros mayores de E. cordifolia, L. sempervirens y $L$. philippiana se originaron de manera vegetativa después del disturbio parcial provocando agrupamiento y una positiva autocorrelación para el d de los árboles en el espacio. Las especies subordinadas no se ven afectadas por la presencia del dosel dominante de $N$. dombeyi. Estos resultados son consistentes con el modelo conceptual propuesto por Zenner (2005) en cuanto a que los disturbios parciales o crónicos provocan una rápida transformación de una estructura simple a una compleja. Esta es una consideración relevante para los bosques nativos de altitudes bajas e intermedias de la zona centro-sur de Chile, los cuales generalmente son afectados por estos disturbios parciales, tales como fuegos de baja intensidad, ramoneo y cortas selectivas.

\section{AGRADECIMIENTOS}

Agradecemos a Horacio Samaniego, Claudio Donoso, y a dos revisores anónimos por sus comentarios y sugerencias, y a Antonio Lara por la base de datos.

\section{LITERATURA CITADA}

ALDRICH PR, GR PARKER, JS WARD \& $\mathrm{CH}$ MICHLER (2003) Spatial dispersion of trees in an old-growth temperate hardwood forests over 60 years of succession. Forest Ecology and Management 180: 475-491.

BAILEY RL \& TR DELL (1973) Quantifying diameter distributions with the Weibull function. Forest Science 19: 97-104.

BAKER PJ, S BUNYAVEJCHEWIN, CD OLIVER \& PS ASHTON (2005) Disturbance history and historical stand dynamics of a seasonal tropical forest in western Thailand. Ecological Monographs 75: 317-343.

BORMAN FH \& GE LIKENS (1981) Patterns and process in a forested ecosystems. SpringerVerlag, New York, NY.

CONNELL JH \& RO SLATYER (1977) Mechanisms of succession in natural communities and their role in community stability and organization. American Naturalist 111: 1119-1144.

COOMES DA \& RB ALLEN (2007) Mortality and treesize distributions in natural mixed-age forests. Journal of Ecology 95: 27-40. 
CHEN J \& GA BRADSHAW (1999) Forest structure in space: A case study of an old-growth spruce-fir forests in Changbaishan Reserve, China. Forest Ecology and Management 120: 219-233.

CHRISTIE DA \& JJ ARMESTO (2003) Regeneration microsites and tree species coexistence in temperate rain forests of Chiloé Island, Chile. Journal of Ecology 91: 776-784.

DÍAZ MF \& JJ ARMESTO (2007) Limitantes físicos y bióticos de la regeneración en matorrales sucesionales de la Isla Grande de Chiloé, Chile. Revista Chilena de Historia Natural 80: 13-26.

DONOSO C (1993) Estructura, variación y dinámica de bosques templados de Chile y Argentina. Ecología Forestal. Editorial Universitaria, Santiago, Chile.

DONOSO C, B ESCOBAR \& J URRUTIA (1985) Estructura y estrategias regenerativas de un bosque virgen de Ulmo (Eucryphia cordifolia Cav.)-Tepa (Laurelia philippiana Phil.) en Chiloé, Chile. Revista Chilena de Historia Natural 58: 171-186.

DONOSO PJ \& CH LUSK (2007) Differential effects of emergent Nothofagus dombeyi on growth and basal area of canopy species in an old-growth temperate rainforest. Journal of Vegetation Science 18: 675-684

DONOSO PJ, C LITTLE, E NEIRA, R REYES, P RUTHERFORD \& C ZAMORANO (2003) Plan de desarrollo integral para el predio Llancahue, Valdivia, Chile. Informe Técnico, Universidad Austral de Chile, Ministerio de Bienes Nacionales, Valdivia, Chile.

DUNCAN RP (1991) Competition and the coexistence of species in a mixed podocarp stand. Journal of Ecology 79: 1073-1084.

FAJARDO A \& P ALABACK (2005) Effects of natural and human disturbances on the dynamics and spatial structure of Nothofagus glauca in southcentral Chile. Journal of Biogeography 32: 18111825.

ESCOBAR B, C DONOSO \& A ZÚÑIGA (2006) Eucryphia cordifolia Cav. En: Donoso C (ed) Las especies arbóreas de los bosques templados de Chile y Argentina. Autoecología: 246-255 Marisa Cuneo ediciones, Valdivia, Chile.

GOFF F \& D WEST (1975) Canopy-understorey interaction effects on trees of the primeval northern hardwood forest. Forest Science 21: 98108.

GUTIÉRREZ AG, JJ ARMESTO \& JC ARAVENA (2004) Disturbance and regeneration dynamics of an oldgrowth North Patagonian rain forest in Chiloé Island, Chile. Journal of Ecology 92: 598-608.

HAASE P (1995) Spatial patterns analysis in ecology based on Ripley's K-function: Introduction and methods of edge correction. Journal of Vegetation Science 6: 575-582.

HE F \& RP DUNCAN (2000) Density-dependent effects on tree survival in an old-growth Douglas fir forests. Journal of Ecology 88: 676-688.

KENKEL NC (1988) Patterns in self-thinning in Jack pine: Testing the random mortality hypothesis. Ecology 69: 1017-1024.

LEPS J \& P KINDLMANN (1987) Models of the development of spatial patterns of an even-aged plant population over time. Ecological Modelling 39: 45-57.

LORIMER CG \& AG KRUG (1983) Diameter distribution in even-aged stands of shade- tolerant and midtolerant tree species. American Midland Naturalist 109: 331-345.

LUSK CH (1995) Seed size, establishment sites and species coexistence in a Chilean rain forest. Journal of Vegetation Science 6: 249-256.

LUSK CH \& A ORTEGA (2003) Vertical structure and basal area development in second-growth Nothofagus stands in Chile. Journal of Applied Ecology 40: 639-645.

MOEUR M (1993) Characterizing spatial patterns of trees using stem-mapped data. Forest Science 39: 756-775.

OLIVER CD \& BC LARSON (1996) Forest stand dynamics. McGraw-Hill, New York, NY.

PERRY GP (2004) SpPack: Spatial point pattern analysis in Excel using Visual Basic for Applications (VBA). Environmental Modelling \& Software 19: 559-569.

PERRY GP, BP MILLER \& NL ENRIGHT (2006) A comparison of methods for the statistical analysis of spatial point patterns in plant ecology. Plant Ecology 187: 59-82.

PICKETT STA, SL COLLINS \& JJ ARMESTO (1987) Models, mechanisms and pathways of succession. Botanical Review 53: 335-371.

RIBEIRO PJ JR \& PJ DIGGLE (2001) geo R: A package for geostatistical analysis. R News 1: 14-18.

R DEVELOPMENT CORE TEAM (2009) R: A language and environmental for statistical computing. Version 2.9.2. URL: http://www.r-project.org. R Foundation for Statistical Computing, Vienna, Austria.

RIPLEY BD (1976) The second-order analysis of stationary processes. Journal of Applied Probability 13: 255-266.

RIPLEY BD (1981) Spatial statistics. John Wiley \& Sons, New York, NY.

ROZAS V (2006) Structural heterogeneity and tree spatial patterns in an old-growth deciduous lowland forests in Cantabria, northern Spain. Plant Ecology 185: 57-72.

ROZAS V \& JJ CAMARERO (2005) Técnicas de análisis espacial de patrones de puntos aplicados en ecología forestal. Investigación Agraria: Sistemas \& Recursos Forestales 14: 79-97.

SALAS C, V LE-MAY, P NÚNEZ, P PACHECO \& A ESPINOSA (2006) Spatial patterns in an oldgrowth Nothofagus obliqua forests in southcentral Chile. Forest Ecology and Management 231: 38-46.

SALAS C \& DP SOTO (2010) Stand variables, Weibull fit and semivariograms for a Nothofagus dombeyi permanent sample plot in Llancahue, Chile Documento Interno No. 1, Proyecto Estudio de Heterogeneidad estructural y espacial de un bosque de Nothofagus dombeyi en Llancahue. Universidad Austral de Chile, Valdivia, Chile.

SALDAÑA A \& CH LUSK (2003) Influencia de las especies del dosel en la disponibilidad de recursos y la regeneración avanzada en un bosque templado lluvioso del sur de Chile. Revista Chilena de Historia Natural 76: 639-650.

SCHABENBERGER O \& FJ PIERCE (2002) Contemporary statistical models for the plant and soil sciences. CRC Press, Boca Raton, FL.

SCHLATTER JE, V GERDING \& H HUBER (1995) Sistema de ordenamiento de la tierra: Herramienta para la planificación forestal aplicado a la X Región. Universidad Austral de Chile, Valdivia, Chile. 
SOTO DP \& PJ DONOSO (2006) Patrones de regeneración en renovales de Drimys winteri en el centro-norte de la Isla de Chiloé: Cambios de acuerdo al tamaño y la densidad relativa. Bosque 27: 241-249.

SOTO DP, PJ DONOSO, D UTEAU \& A ZUÑIGAFEEST (2009) Environmental factors affect the spatial arrangement of survival and damage in a outplanted Nothofagus dombeyi plantation seedlings in the Chilean Andes. Interciencia 34: 100-105.

SOTO DP \& H FIGUEROA (2008) Efectos de las alteraciones antrópicas sobre la estructura y composición de rodales de Pilgerodendron uviferum en la Cordillera de la Costa de Chile. Ecología Austral 18: 13-25.

STOKES MA \& TL SMILEY (1968) An introduction to tree-ring dating. University of Chicago Press, Chicago, IL.

SZWAGRZYK J \& M CZERWCZAK (1993) Spatial patterns of trees in natural forests of east-central Europe. Journal of Vegetation Science 4: 469476.

VEBLEN TT \& D ASHTON (1978) Catastrophic influences on the vegetation of the Valdivian Andes. Vegetatio 36: 149-167.

VEBLEN TT, FM SCHLEGEL \& B ESCOBAR (1980) Structure and dynamics of old-growth
Nothofagus forests in the Valdivian Andes, Chile. Journal of Ecology 68: 1-31.

VEBLEN TT, C DONOSO, FM SCHLEGEL \& B ESCOBAR (1981) Forest dynamics in SouthCentral Chile. Journal of Biogeography 8: 211247.

VEBLEN TT, T KITZBERGER \& R VILLALBA (2004) Nuevos paradigmas en ecología y su influencia sobre el conocimiento de la dinámica de los bosques del sur de Argentina y Chile. En: Arturi MF, JL Frangi \& JF Goya (eds) Ecología y manejo de los bosques nativos de Argentina: 148. Editorial Universidad Nacional de la Plata, La Plata, Argentina.

WALLER LA \& CA GOTWAY (2004) Applied spatial statistics for public health data. John Wiley \& Sons, Hoboken, NJ, USA.

WANG X, Z HAO, J ZHANG, J LIAN, L BUHANG, J YE \& X YAO (2008) Tree size distribution in an oldgrowth forest. Oikos 118: 25-36.

WARD JS, GR PARKER \& FJ FERRANDINO (1996) Long-term spatial dynamics in an old-growth deciduous forest. Forest Ecology and Management 83: 189-202.

ZENNER EK (2005) Development of tree size distribution in Douglass-fir forests under differing disturbances regimes. Ecological Applications 15: 701-714.

Editor Asociado: Mauricio Lima

Recibido el 18 de mayo de 2009; aceptado el 5 de abril de 2010 
